\title{
Bubble collapse as the source of tremor at Old Faithful
}

\section{Geyser}

\author{
Sharon Kedar and Hiroo Kanamori \\ Seismological Laboratory, California Institute of Technology, Pasadena \\ Bradford Sturtevant \\ Graduate Aeronautics Laboratories, California Institute of Technology, Pasadena
}

\begin{abstract}
Old Faithful Geyser, Yellowstone, was used as a natural laboratory for fluid-flow-induced seismic activity. Pressure measurements within the geyser's water column, obtained simultaneously with seismic measurements on the surface, demonstrated that the tremor observed at Old Faithful results from impulsive events in the geyser. Tremor intensity is controlled by the rate of occurrence of these impulsive events. There is no resonance observed within the water column. The impulsive events are modeled by a collapse of a spherical bubble, including the effects of residual non-condensible gas and damping. The pressure data can be explained by a collapse of a $\sim 5 \mathrm{~cm}$ radius bubble driven by a pressure difference of $\Delta P=0.3 \times 10^{5} \mathrm{~Pa}$ and effective viscosity $\nu_{E}=0.04 \mathrm{~m}^{2} / \mathrm{s}$. Using a quasi-static geyser model, we treat the individual bubble collapses as cooling events that occur when the water column reaches a critical temperature. Their rate of occurrence is controlled by the heating rate $d T / d t$ of the water column. As a result, the intensity of the hydrothermal and seismic activities is determined by the heat and mass input rate into the geyser. It is demonstrated that a sharp widening of the conduit can cause the number of events per unit time to drop (as observed) while the water level is still rising and heat is being input, and thus the tremor intensity can be modulated by variations in the conduit shape.
\end{abstract}

\section{Introduction}

Old Faithful is probably the most studied geyser in the world. Located in the Upper Geyser Basin of Yellowstone National Park, its surface expression is a $4 \mathrm{~m}$ high, $60 \mathrm{~m}$ wide mound with an approximately $2 \mathrm{~m} \times 1$ $m$ opening at the top. The conduit extends downward, successively narrowing and opening into larger spaces, as described by Birch and Kennedy [1972] and as inferred from the spectacular video recordings made by Hutchinson et al. [1997]. The geyser's eruptions are 25 min long with intervals between them ranging from 30 to $100 \mathrm{~min}$, and their time of occurrence is predictable to within $15 \mathrm{~min}$.

J. Rinehart was the first to deploy seismometers around the geyser, as well as to measure temperatures inside it [Rinehart, 1965, 1967, 1980]. temperature inside it. Birch and Kennedy [1972] continued with temperature measurements at different depths in the geyser, and Kieffer [1984] gave an elaborate description of the geyser's behavior including its seismicity and

Copyright 1998 by the American Geophysical Union.

Paper number 98JB01824.

0148-0227/98/98JB-01824\$09.00 thermodynamics. Kieffer [1984] first pointed out the similarities between geyser seismicity and volcanic seismicity and the possible relevance of geyser studies to the interpretation of volcanic tremor, the nonearthquake signals which precede and accompany volcanic activity.

These studies provided a good overall understanding of the geyser's behavior. However, since all past studies used seismometers without simultaneous time-resolved measurements of pressure and temperature, the source of tremor and its interaction with the solid medium at Old Faithful were not fully understood.

Our work at Old Faithful was designed to establish a cause-and-effect relationship between the source of seismic noise and the observed tremor. We have carried out three seismic studies at Old Faithful Geyser (field seasons 1991, 1992, and 1994), in which time-resolved pressure variations inside the geyser, passive seismicity around the geyser, and seismic response to an external sledge hammer source have been measured. Kedar et al. [1996] showed that the seismic activity between eruptions at Old Faithful Geyser is composed of a superposition of discrete events originated by pressure pulses inside Old Faithful, in analogy to volcanic long-period (LP) events, which are considered the building blocks of continuous volcanic tremor. The seismic observations, 


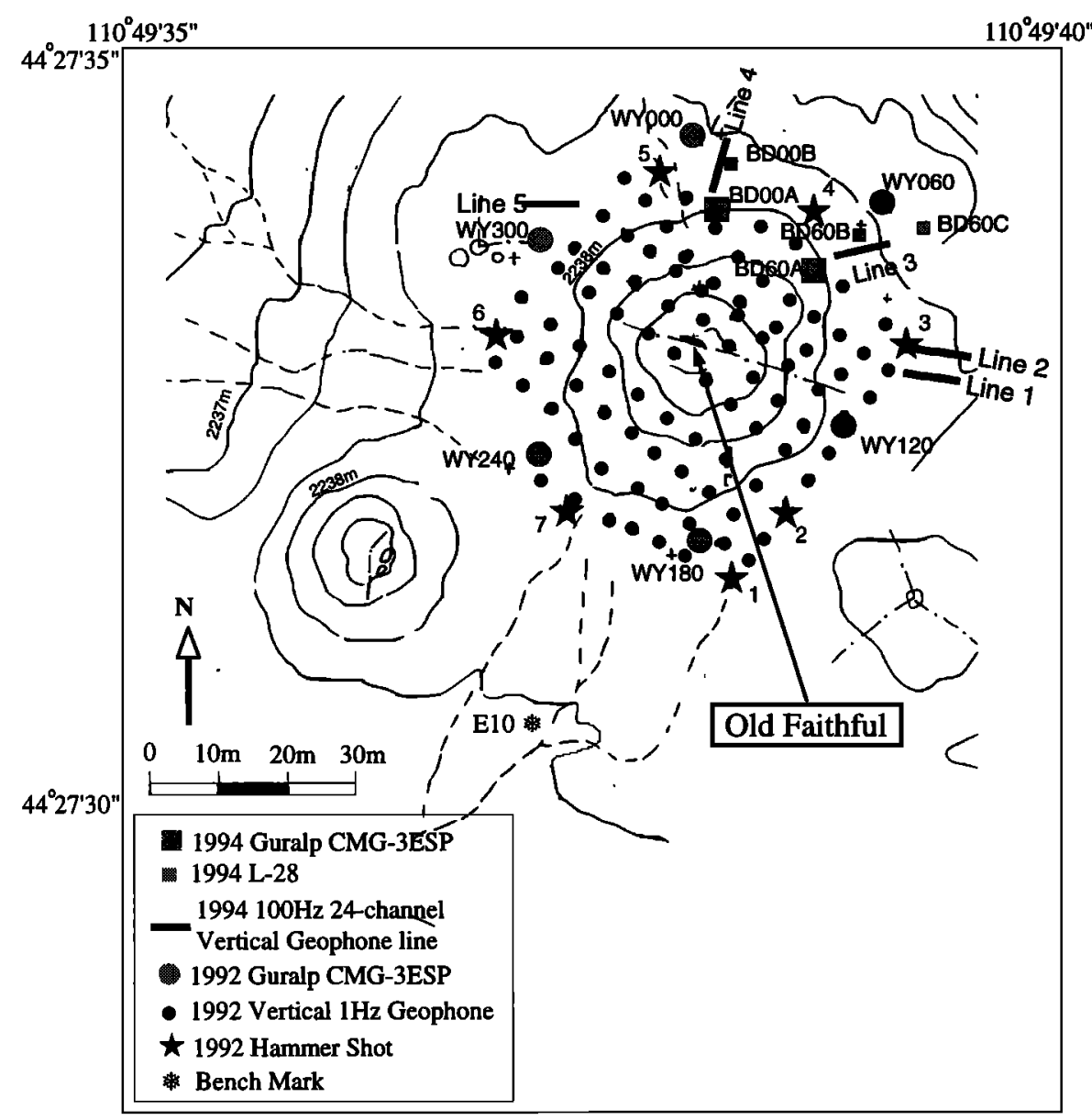

Figure 1. A map of the survey area and instrument setups for the 1992 and 1994 deployments in relation to Old Faithful and two of its neighboring passive domes. The orientation of the geyser's edifice is indicated by the dashed line across its dome.

which were made with both broadband and short-period instruments, were used by Kedar et al. [1996] to demonstrate that the harmonic characteristics of the seismicity could result from reverberations in a near-surface soft layer. This paper is a continuation of the paper by Kedar et al. [1996] and concentrates on the physical processes within Old Faithful's water column.

\section{Data}

\subsection{Fieldwork and Setup}

Three scientific excursions to Old Faithful Geyser were made (Figure 1). The first (not shown in Figure 1) was carried out in 1991 and deployed two broadband sensors (GURALP CMG-3ESP, flat velocity response 0.0333-50 Hz) and REF-TEK six-channel portable data loggers with 16-bit digitizers. This served as a pilot study for a second and a more elaborate seismic survey in October 1992, which incorporated broadband recordings of both geyser-generated signals and sledge hammer pulses. This survey included six broadband setups identical to the 1991 instruments. These were placed around the geyser in different configurations for continuous recordings at 100 and 200 samples per sec- ond for periods of a day at a time. In addition, an array of 96 short-period (natural frequency $1 \mathrm{~Hz}$ ) vertical geophones were placed in a tight grid over the geyser's dome. The third field experiment (in October 1994) involved the simultaneous deployment of two three-component broadband sensors, three L-28 threecomponent short-period sensors (natural frequency 4.5 $\mathrm{Hz}$ ), and a probe designed to measure pressure inside the water column. All three sensor types were recording simultaneously and continuously at 250 samples per second.

\subsection{Eruption Cycle}

Figure 2 displays 6-hour-long unfiltered vertical and north-south velocity records at station WY000 (Figure 1). These records include several eruption cycles (defined as the time interval between the end of one eruption to the end of the next). The general pattern of the eruption cycle starts with a quiet period, of $1 / 2$ hour duration. Seismicity then increases in intensity and amplitude and then gradually decays until the final moments before an eruption. A short ( $50 \mathrm{~min})$ eruption cycle shows a similar behavior without the initial period of seismic silence. Kieffer [1984] explained the am- 


\section{WY000 10/21/92}

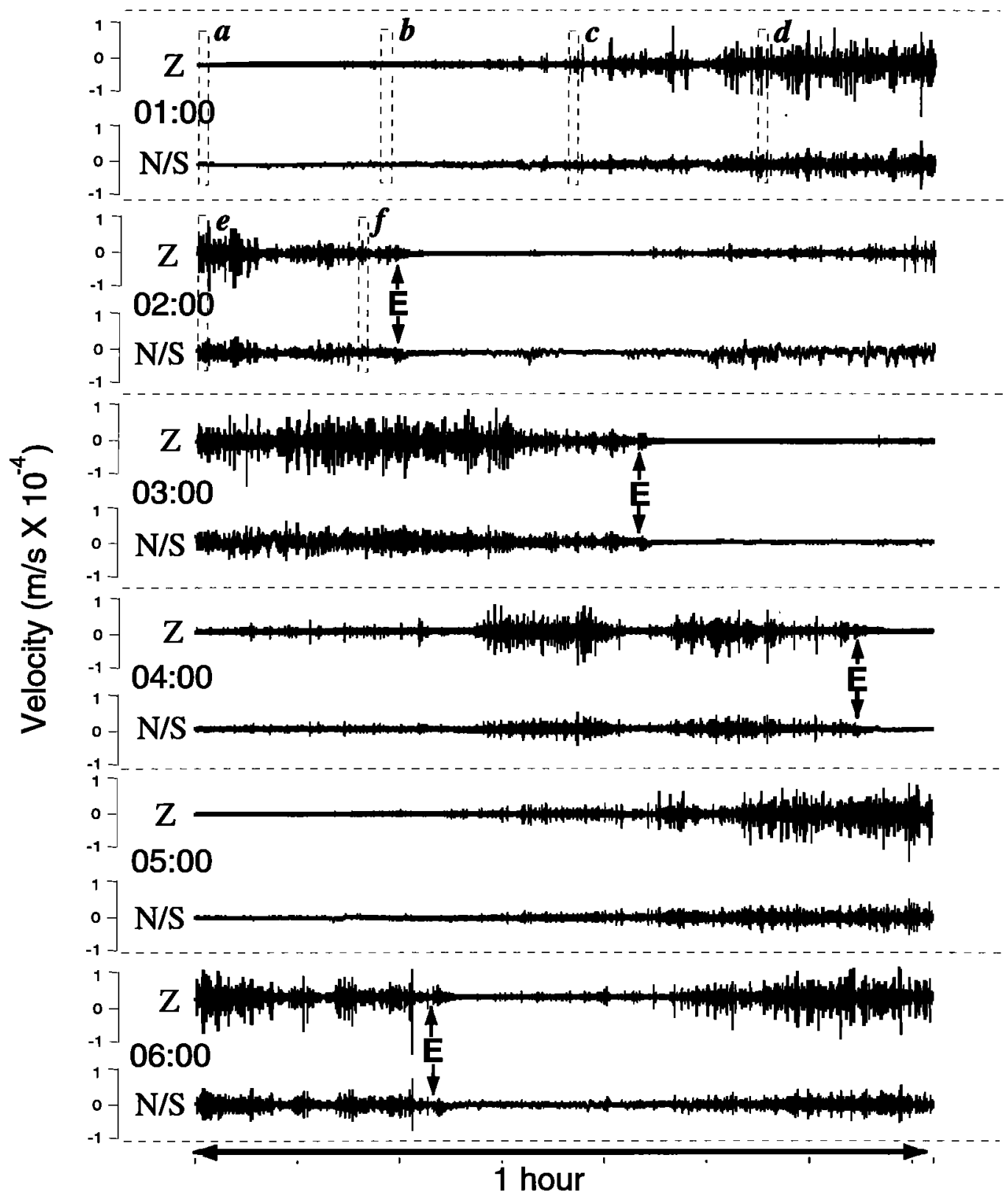

Figure 2. Vertical and N/S (radial) components for 6 hours at station WY000. Eruptions are marked by E. Six 1-min time windows marked by $a-f$ are displayed in Figures 3.

plitude decay by an increased acoustic impedance mismatch between a two-phase water-steam mixture and the conduit walls due to an increased amount of steam in the final stages of the eruption cycle. Although the interval between eruptions fluctuates over a period of several years, it is known to have a bimodal distribution with peaks at $\sim 50 \mathrm{~min}$ and $\sim 75 \mathrm{~min}$ [Rinehart, 1980].

2.2.1. Discrete events. Figure 3 demonstrates that the seismic activity depicted in Figure 2 is composed of discrete events with a sharp onset and a characteristic decay time. It is evident that the intensity of the activity is dependent on the rate of occurrence of these discrete events as well as their amplitude and that the pulses get stronger and more frequent as the eruption cycle progresses. As shown in Figure 3, in the early stages of the eruption cycle infrequent low-amplitude pulses are observed. The pulses get stronger and more frequent until their amplitude and rate become steady. Minutes before the eruption the amplitude decreases due to the poor acoustic impedance match between the bubbly fluid and the geyser wall [Kieffer, 1984], while the rate stays fairly constant.

2.2.2. Event rate. A quantitative analysis of the number of events per minute (Figure 4) illustrates the overall seismic behavior described above. The events 

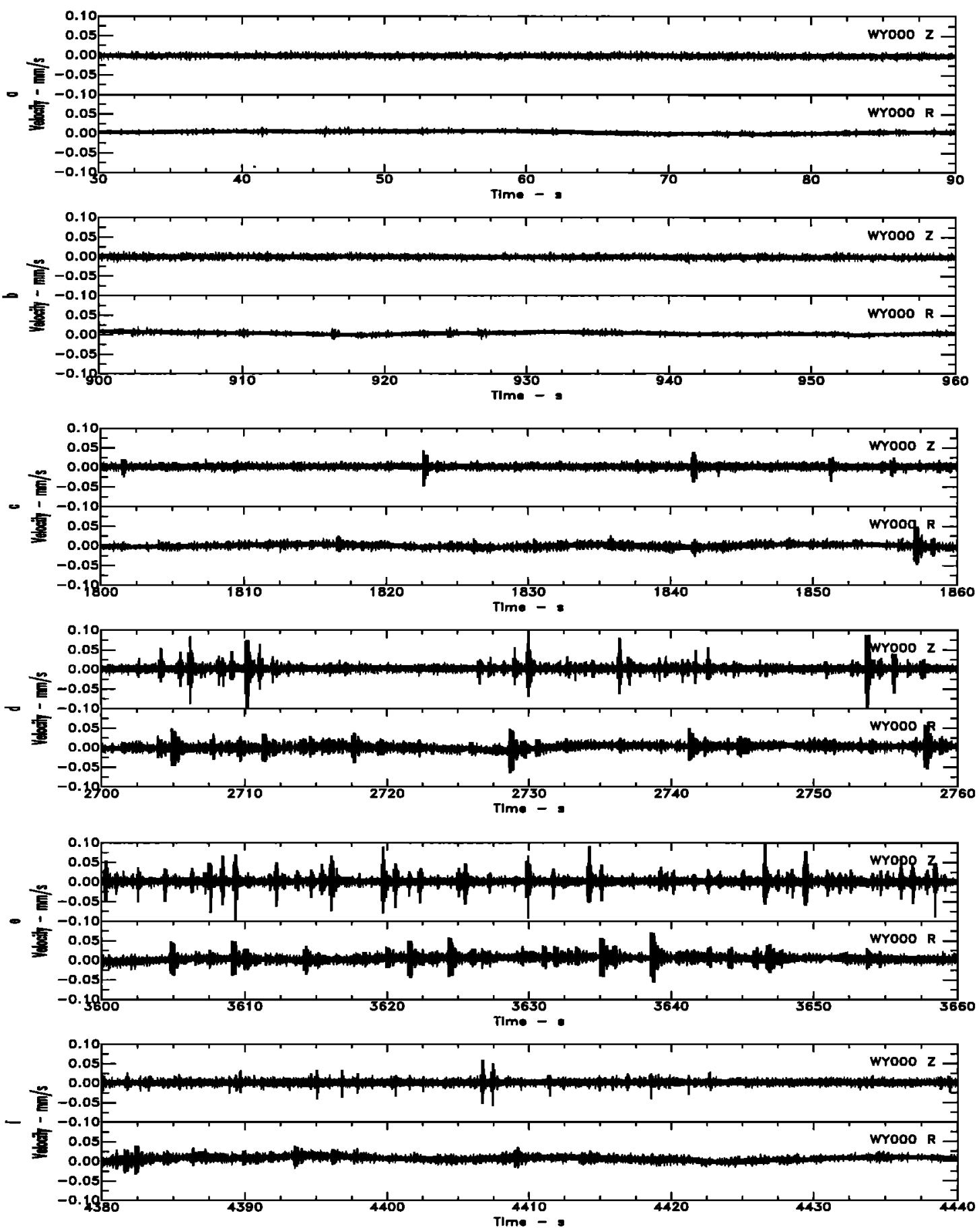

Figure 3. An enlargement of the 1-min time windows marked $a-f$ in Figure 2 .

were counted by a computerized counter designed to detect events energetic enough to appear distinctly above the noise level (signal to noise $\sim 10$ ). The eruption itself generates a distinct but weak seismic signal as can be observed in Figures 2 and 5 . The eruption signal is characterized by a 1-3 min long coda generated mainly by the ejected water falling on the ground.

It is apparent from Figure 4 that the number of events per minute increases over a time scale of about $20 \mathrm{~min}$, then decreases suddenly, then increases again to plateau for another 30-40 min. We will describe a model which accounts for the general behavior observed, namely, the initial rise in event rate, the sudden decrease, and the final plateau as depicted by dashed lines in Figure 4.

2.2.3. The eruption. Although we will focus on the activity between eruptions in this paper, here we briefly describe the eruptions themselves. The seismic signals from several eruptions are presented in Figure 5. An eruption occurs when a mass of water is removed temporarily from the top of the superheated column, 


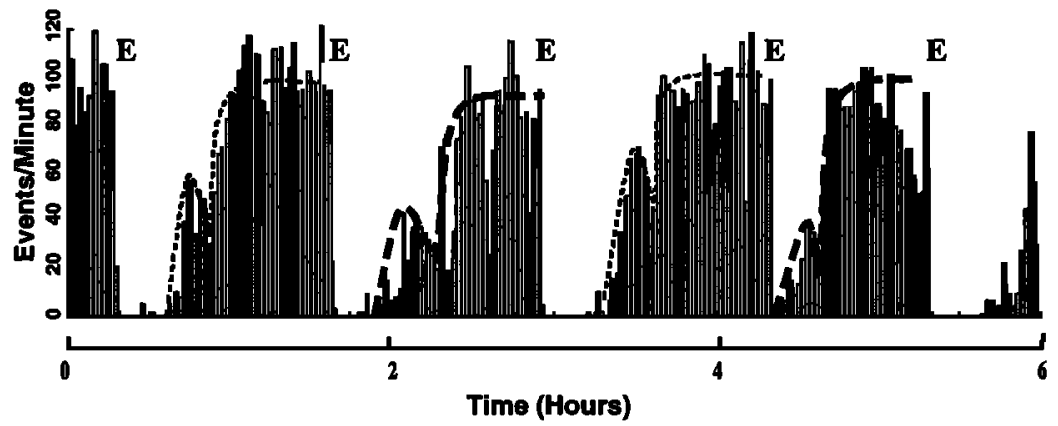

Figure 4. Event rate at WY000 inferred from a 12-hour long record. The general trend to be modeled is outlined by a dotted line. Eruptions are marked by " $\mathrm{E}$." The drop in event rate toward the end of the cycle is an artifact of the automated event counter, because the signal-to-noise ratio decreases as the eruption nears.
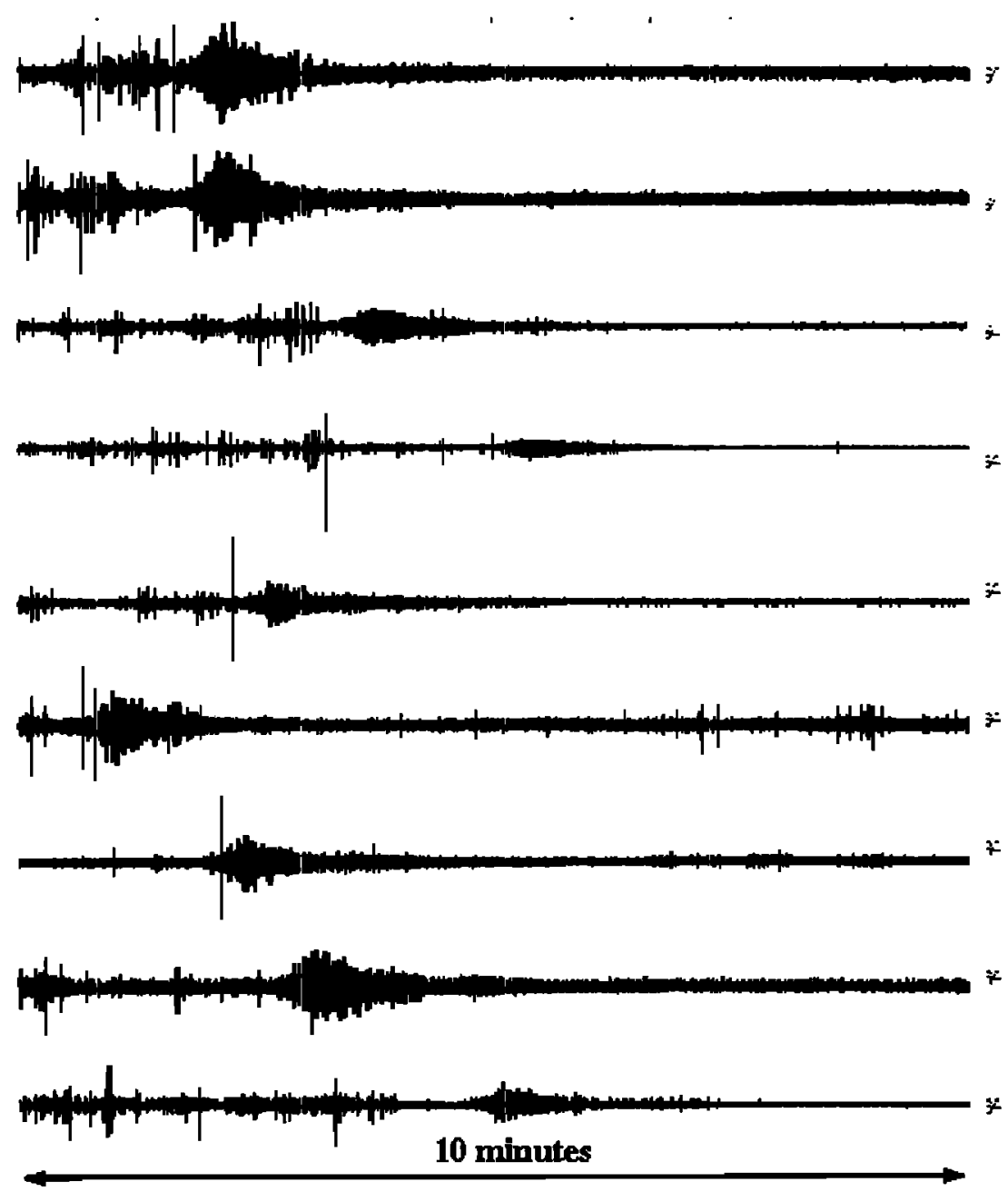

Figure 5. A 10-min window of the vertical component at WY000 around nine eruptions. In some cases (four out of the nine eruptions) there is a distinct event which seems to initiate the eruption. The significance of this observation is debatable, as it appears only for some eruptions. There is no other signal which may be used as a prediction tool, other than the overall reduction in the background noise amplitude in the minutes prior to an eruption. In most cases, the eruption's seismic signal emerges from the background noise with no clear precursor. 


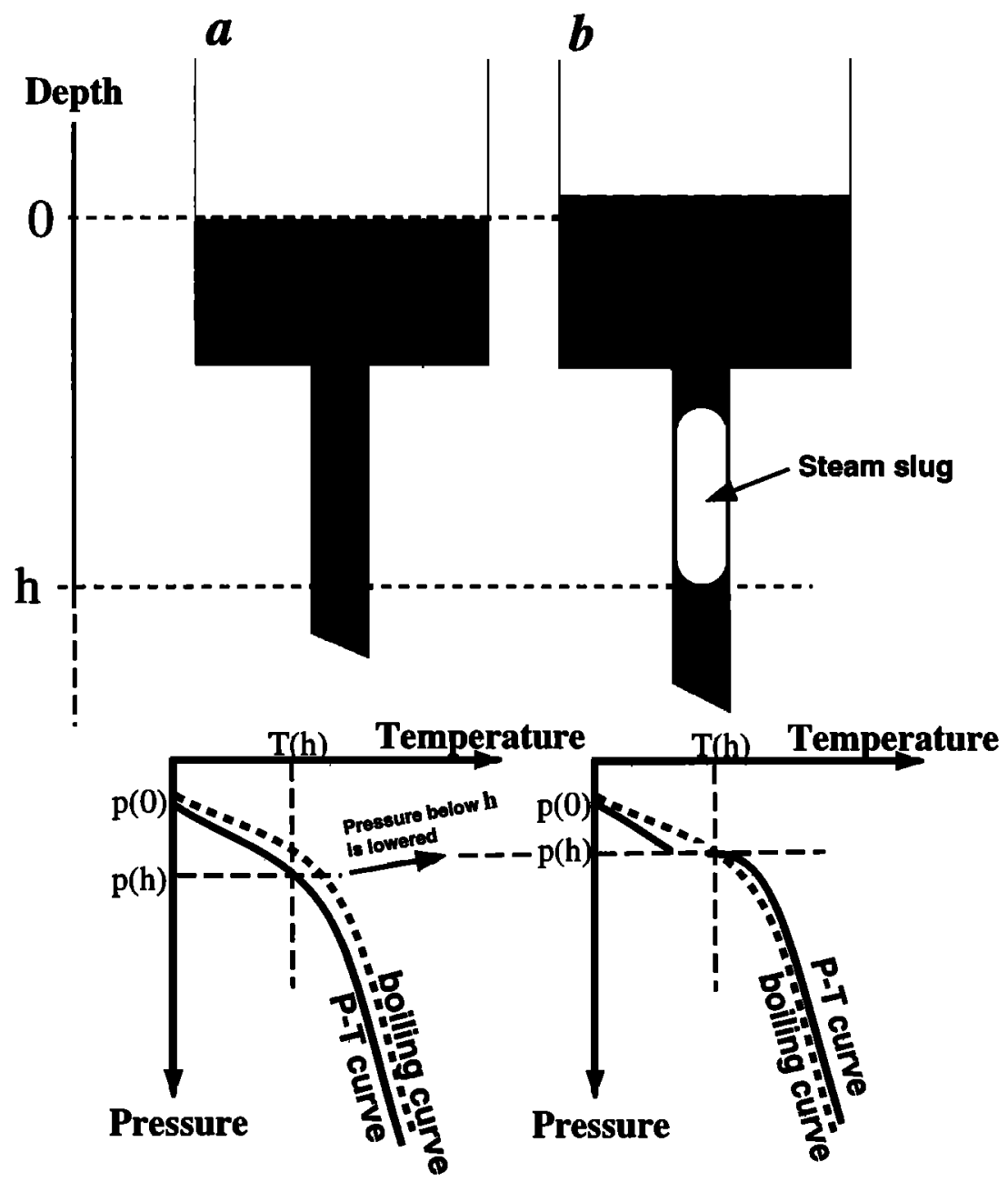

Figure 6. A schematic illustration of the process of instantaneous boiling of a water column as a result of a formation of a steam slug. As a slug of steam is formed inside the pipe, the water is pushed up into the tank. Since the mass of the steam slug is negligible, most of the water which occupied the volume (now occupied by vapor) is spread over a wider space, thus lowering the hydrostatic pressure in the fluid below the slug at depth $h$. If the fluid is near boiling, a slight reduction of the hydrostatic pressure will cause instantaneous boiling of the fluid below the slug. It should be pointed out that the same phenomenon takes place for any shape of widening pipe (such as a funnel).

causing a sufficient drop in the hydrostatic head to result in instantaneous boiling of the entire column and subsequent evacuation of the conduit in the form of an eruption. Two main mechanisms may be responsible for the reduction in the hydrostatic head; a temporary displacement of a mass of water by an explosive process, or a mass of water pushed into a larger diameter space at the top of the water column due to volume change. The former is known as "preplay" [Rinehart, 1980], a process observed 5-10 min before an eruption at Old Faithful, which starts like a normal eruption that immediately dies as the displaced water falls back into the conduit. The latter occurs when a slug of steam in a conduit pipe displaces a volume of water upwards into a larger diameter space (Figure 6), thus lowering the hydrostatic pressure in the fluid below the slug and causing instantaneous boiling [Griffith, 1962].

\subsection{Pressure Measurements}

Based on the observations from 1991 and 1992, a probe was designed and built to measure pressure inside the water column simultaneously with seismic measurements on the geyser's dome. The instrument consisted of three sensors $3 \mathrm{~m}$ apart on a coaxial cable, each containing a KULITE XTC-190, high-frequency pressure transducer (Figure 7). In October 1994 the instrument was lowered into the geyser's conduit during the quiet period in a long eruption cycle until the bottom sensor was $22 \mathrm{~m}$ deep and was below $7 \mathrm{~m}$ of water.

Figure 8 displays the $\sim 30$-min-long pressure data recorded inside the conduit of Old Faithful. A question could be raised about the possible depth drift of the sensors in the turbulent water column. From Figure 8 , it is apparent that fluctuations in the hydrostatic 

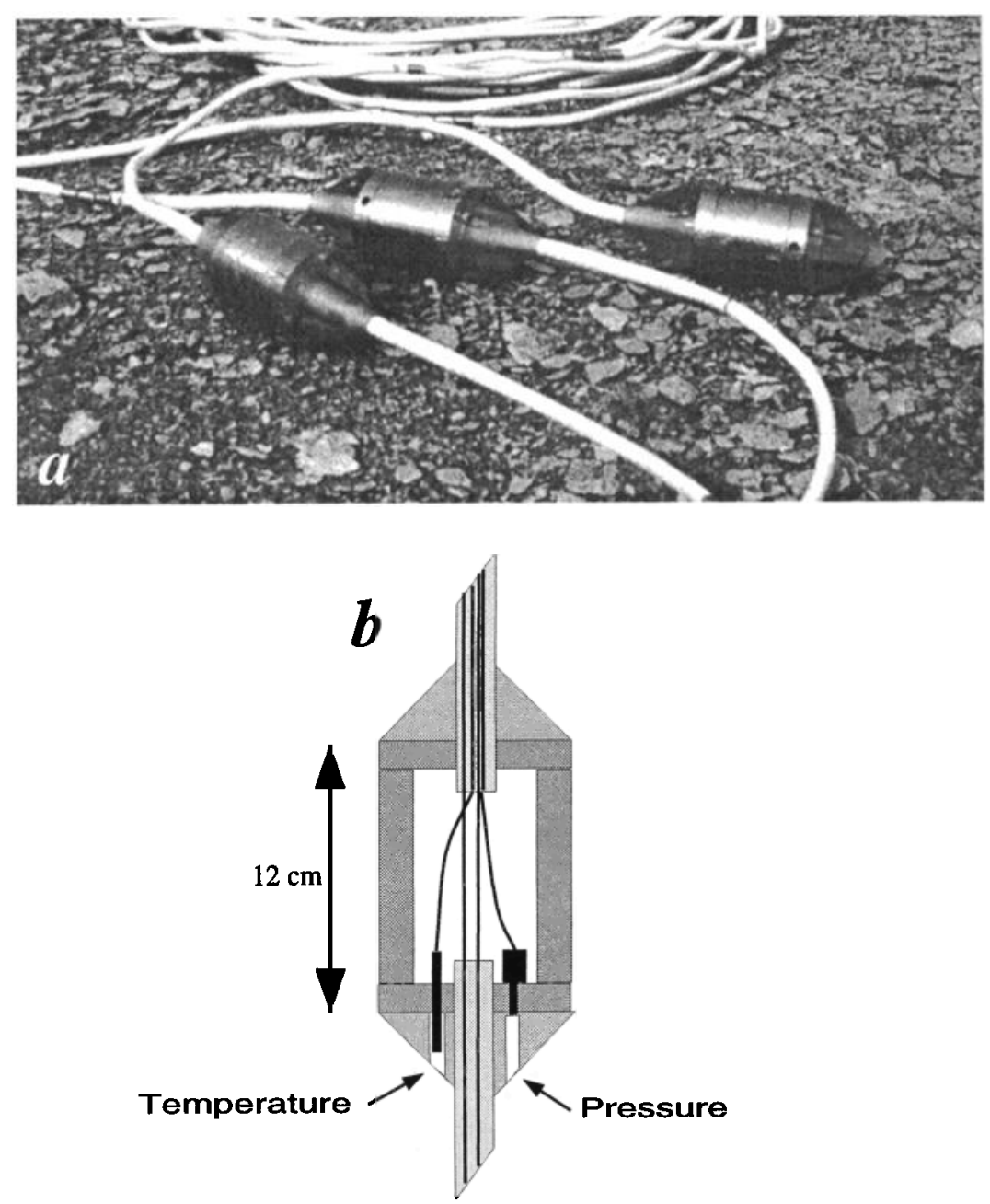

Figure 7. (a) The three pressure sensors laying on sinter deposits at the foot of Old Faithful's dome. (b) A schematic diagram of the interior of the containers. The thermocouple record was too noisy to be used for analysis.

pressure measured by the pressure transducers are observed. However, the overall trend of hydrostatic head increase is consistent with Old Faithful's filling history [Hutchinson et al., 1997], and since we will be focusing on the short-period discrete events that compose the pressure activity, any possible depth fluctuations become negligible.

The malfunctioning of the bottom transducer is attributed to water penetrating into its container; thus its record will be ignored. However, after a period of thermal adjustment, the top and middle transducers display a behavior consistent with their relative locations in the water and a steady rise in the pressure over a period of 30 minutes as the geyser is filling.

Figure 9 displays a 2-s-long pressure and seismic records (station $\mathrm{BD} 60 \mathrm{~A}$ ) during which a single pressure pulse in the water is followed $\sim 0.1 \mathrm{~s}$ later by a seismic signal. This observation (which is representative of all the geyser-generated activity at Old Faithful Geyser as was demonstrated by Kedar et al. [1996]) establishes a cause-and-effect relationship between a pressure pulse taking place within the water column and the seismic signal that follows it. Therefore the seismic activity's intensity (i.e., the number of seismic events per unit time) is determined by the rate of occurrence of individual pressure pulses. These pressure pulses are generated near the top of the water column and are strongly attenuated within it, while no pressure reverberations are observed within the water column. This eliminates any notion of "organ-pipe" type resonance in the water column as the source of tremor in the case of Old Faithful Geyser.

\section{The Source}

In section 2 it was established that tremor at Old Faithful originates from sharp pressure pulses within the water column. In order to gain further physical understanding of the nature of these processes, two problems are posed:

1. What are the characteristics (i.e., shape, timescale, and amplitude) of the impulsive source? 


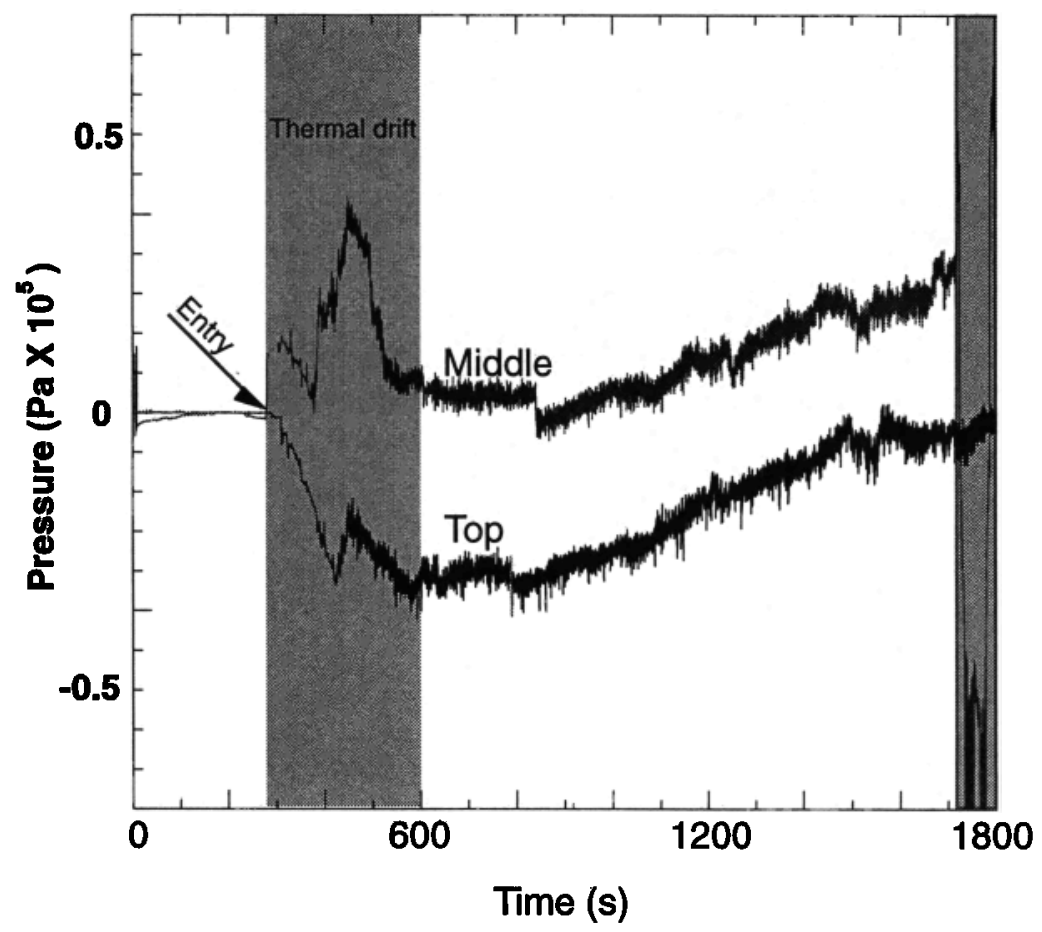

Figure 8. The long-period behavior of the pressure measured inside the conduit. The top and middle transducers are $3 \mathrm{~m}$ apart; thus the top transducer is measuring a $\sim 3.5 \times 10^{4} \mathrm{~Pa}$ lower pressure than the middle transducer. The bottom transducer is malfunctioning due to water leaking into the container and is not shown here. The sensors were put in immediately after a long eruption and were taken out $\sim 40 \mathrm{~min}$ later, prior to the following eruption. The first 30 min are presented here, until the middle transducer malfunctioned. After about 5 min of drift due to thermal effects, the pressure sensors stablize. The zero pressure value is with reference to the middle transducer.

2. What controls the rate of occurrence of pulses? The rate of occurrence of pulses determines whether continuous or impulsive tremor is excited. The filling rates and heating rates, the interaction between them, and their effect on the rate of pressure pulse excitation will be discussed.

\subsection{Source of Impulses}

A common source of repetitive pressure impulses in a column of boiling water is bubble collapse [Blake, 1986; Boure et al., 1972], as was suggested by Kieffer [1984] and Kedar et al. [1996] in the context of Old Faithful. Bubble collapse was also suggested as the source of volcanic tremor by Leet [1988]. When a steam bubble rises to some cooler, lower-pressure zone in the water column, the steam vapor condenses and the bubble collapses, generating a pressure pulse with a characteristic collapse time $\tau_{c}$. Sometimes a rebound or several rebounds result from an incomplete collapse of the bubble, as is shown in Figure 10. This is frequently observed in the laboratory [Blake, 1986]. Similarly, when a large bubble of the order of the size of the conduit bursts at the water surface, the displaced mass of water will drop back on the emptied cavity (a process sometimes referred to as "chugging" [Boure et al., 1972]), gener- ating a pulse. The collapse time $\tau_{c}$ is measured from the moment the pressure starts increasing to the time of the pressure peak as is demonstrated in Figure 10. The following discussion will concentrate on attempting to model the recorded pressure pulses as a bubble collapse.

3.1.1. Rayleigh collapse. The simplest collapse model was calculated by Rayleigh [1917], who estimated the collapse time of an empty spherical cavity in a body of incompressible fluid with constant pressure $P_{\infty}$ at infinity. The equation of motion in spherical coordinates is then

$$
\ddot{R} R+\frac{3}{2}(\dot{R})^{2}=\frac{P(R)-P_{\infty}}{\rho}=-\frac{\Delta P}{\rho}
$$

where $R$ is the bubble radius, $\rho$ is the fluid density, and $\dot{R}=d R / d t$. For a spherical void the wall pressure $P(R)=0$ and the collapse time is

$$
\tau_{c}=0.915 R_{0} \sqrt{\rho / P_{\infty}}
$$

where $R_{0}$ is the initial (maximum) bubble radius. Choosing the driving pressure $P_{\infty}$ to be atmospheric pressure, $10^{5} \mathrm{~Pa}$, and the density of water, $\rho=1000 \mathrm{~kg} / \mathrm{m}^{3}$, gives for the average observed collapse time of $\tau_{c}=0.05 \pm 0.02$ 


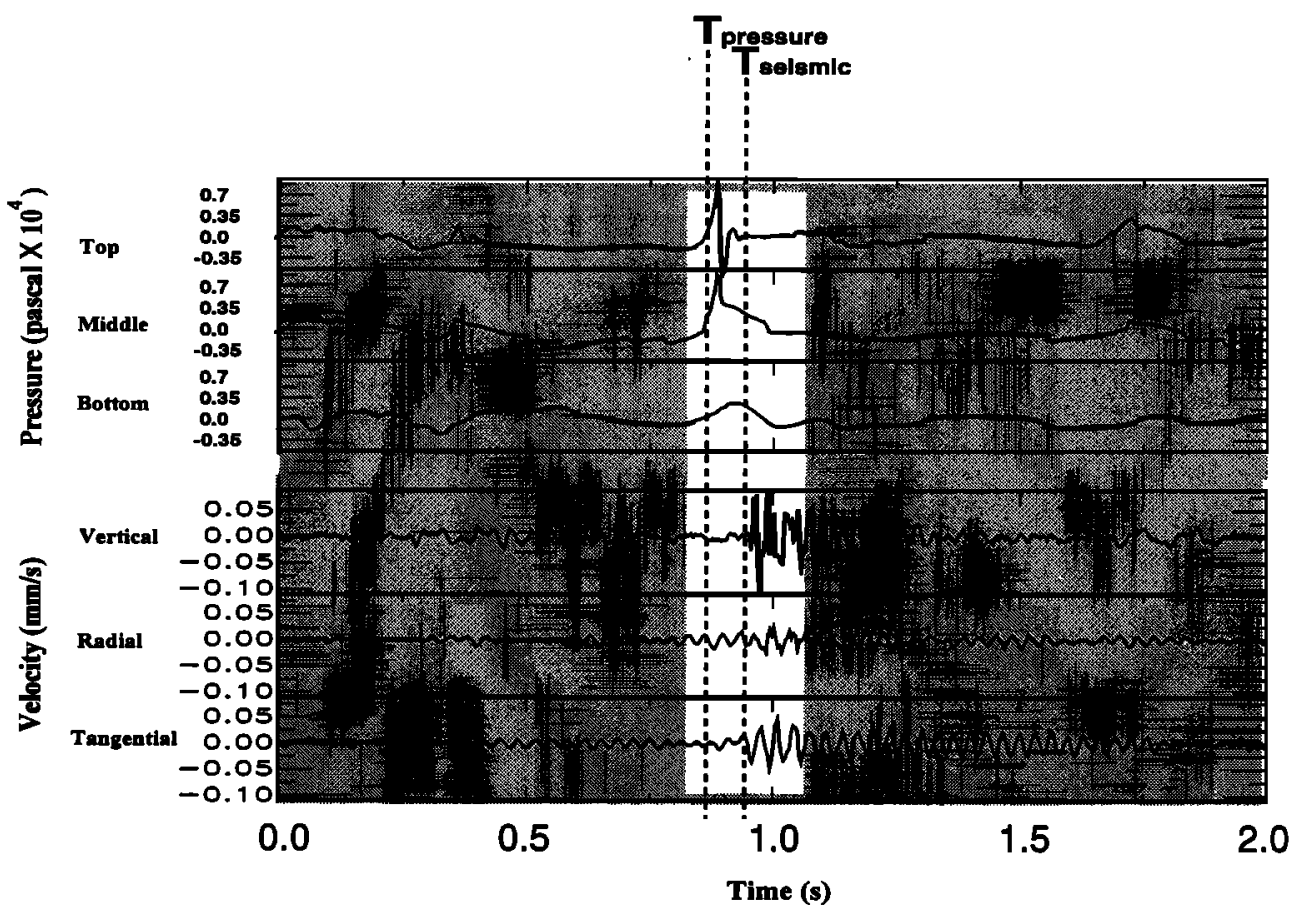

Figure 9. A simultaneous record of pressure and seismic waves shows a distinct cause-andeffect relationship between the impulsive pressure source and the impulse response of the rock surrounding the water column [also see Kedar et al., 1996]. In addition, the pressure pulse, which is strongest at the top transducer, strongly attenuates downward, while no pressure reverberations are apparent within the water column.

$\mathrm{s}$ a bubble radius of $R_{0} \sim 0.5 \mathrm{~m}$. This result is clearly not applicable to Old Faithful since for the most part the geyser conduit is much narrower than $1 \mathrm{~m}$, and at its narrowest point, it is only $\sim 0.1 \mathrm{~m}$ wide. Therefore a more complicated model needs to be explored, which includes processes that yield smaller bubble radii from the observed pressure amplitudes.

3.1.2. The role of noncondensible gases and damping. The Rayleigh collapse is an idealized case. In reality, noncondensible gas such as $\mathrm{CO}_{2}$ in water, which is trapped inside the bubble, is acting as a spring as the gas is compressed during the collapse phase. As can be seen from Figure 10, two to three collapse cycles are commonly observed in the laboratory as well as at Old Faithful. As is also observed, damping plays an important role in the collapse process and is incorporated into the calculation through an additional viscous term. The above physical processes are described by the Rayleigh-Plesset equation [Plesset, 1949]

$$
\ddot{R} R+\frac{3}{2}(\dot{R})^{2}=\frac{1}{\rho}\left[P_{v}+P_{g_{0}}\left(\frac{R_{0}}{R}\right)^{3 \gamma}-P_{\infty}\right]-\frac{4 \nu}{R} \dot{R}
$$

where $P_{v}$ is the vapor pressure of condensible gases inside the bubble, $P_{g 0}$ is the initial noncondensible gas pressure, $\gamma$ is the specific heats ratio, and $\nu$ is the kinematic viscosity of the surrounding fluid. The last term on the right-hand side is the damping term.
When $\nu=0$, equation (3) results in nonlinear oscillations, while in the limit of small oscillations, it yields linear harmonic oscillations:

$$
\ddot{R} R_{0}=\frac{1}{\rho}\left[P_{v}+P_{g_{0}}\left(1+3 \gamma \frac{R_{0}-R}{R_{0}}\right)-P_{\infty}\right]
$$

When $P_{v}=P_{g_{0}}=0$ and $\nu=0$, equation (3) yields Rayleigh collapse (equation (1) ). For water at $\sim 100$ ${ }^{\circ} \mathrm{C}, \nu=10^{-6} \mathrm{~m}^{2} / \mathrm{s}$, resulting in a negligible damping term, since the minimum radius is never small enough to increase the damping force to the magnitude of the pressure or inertia forces. Nonetheless, damping does occur, and some other damping mechanism needs to be pursued.

The calculation based on equation ( 3 ) is shown in Figure 11 , where an effective viscosity $\nu_{E}=0.04 \mathrm{~m}^{2} / \mathrm{s}$ and an isothermal process $(\gamma=1)$ yield a reasonable bubble radius $(5.5 \mathrm{~cm})$ and a good fit to the data from the onset of collapse. (It should be noted that using $\gamma=1.4$ does not significantly change the bubble dimensions.) The model successfully describes the general characteristics of the process, namely, the period of oscillation and the overall damping. However, the effective viscosity is much higher than the viscosity of the water, implying that some other mechanism is responsible for the damping.

As was shown by Chapman and Plesset [1971], at large bubble radii, the effective viscosity is significantly 

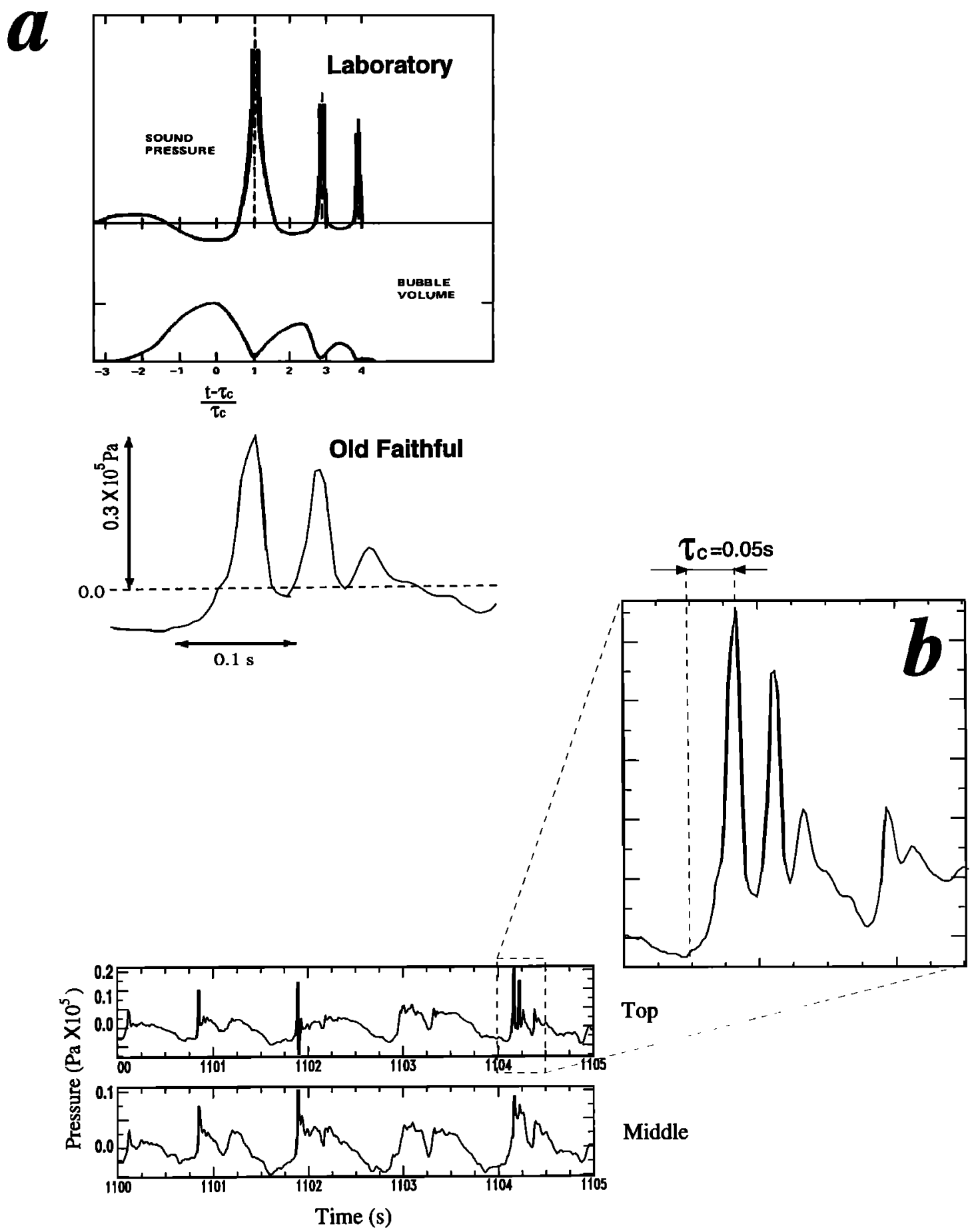

Figure 10. (a) Characteristics of bubble collapse. Comparison between a laboratory measurement [Blake, 1986] and (b) the pressure pulse. The collapse time $\tau_{c}$ is measured from the moment the pressure starts increasing to the time of the pressure peak.

larger than the liquid viscosity due to energy lost by acoustic radiation and thermal diffusion. Therefore

$$
\nu_{E}=\nu_{l}+\nu_{A}+\nu_{T}
$$

where $\nu_{E}$ is the effective viscosity and $\nu_{l}, \nu_{A}$, and $\nu_{T}$ are the liquid, acoustic, and thermal viscosities, respectively. It follows that at radii of a few centimeters for water-air mixtures the acoustic viscosity dominates
[Chapman and Plesset, 1971], and for small oscillations, it is

$$
\nu_{A}=\frac{f^{2} R_{0}^{3}}{4 c_{l}}
$$

where $f$ is the bubble oscillation frequency and $c_{l}$ is the speed of sound in the liquid. For the observed frequency of $f=1 / \tau_{c} \sim 20 \mathrm{~Hz}$ with $c_{l}=1500 \mathrm{~m} / \mathrm{s}$ and a collapse radius of $0.05 \mathrm{~m}, \nu_{A} \sim \nu_{E} \sim 10^{-5} \mathrm{~m}^{2} / \mathrm{s}, 3$ orders of 


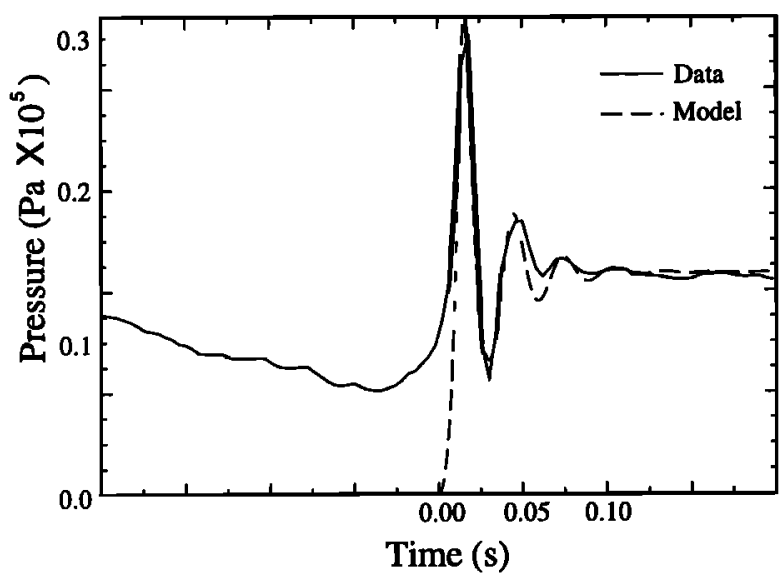

Figure 11. Time series of a collapse of a bubble with an initial radius $R_{0}=5.5 \mathrm{~cm}$, internal pressure $P_{g_{0}}=$ $0.2 \times 10^{5} \mathrm{~Pa}$, with a driving pressure $\Delta P=0.3 \times 10^{5} \mathrm{~Pa}$ and $\nu_{E}=0.04 \mathrm{~m}^{2} / \mathrm{s}$. The driving pressure is equivalent to the weight of $3 \mathrm{~m}$ of water. Note that the zero level was chosen arbitrarily, since only the collapse process (not the inflation) is illustrated here.

magnitude smaller than the viscosity used in Figure 11. If the region of collapse contains $10^{-3}$ mass fraction of steam, $c_{l}$ can be as low as $20 \mathrm{~m} / \mathrm{s}$ [Kieffer, 1977], and then $\nu_{E} \sim 10^{-3} \mathrm{~m}^{2} / \mathrm{s}$, closer to the observed value but still an order of magnitude too small. Therefore we must conclude that mechanisms other than acoustic, thermal, or viscous damping are required to explain the strong damping observed.

A hint for the possible dissipation mechanism may be found in the large attenuation in the water column (Figure 9). Analysis of arrival times of the pressure pulses at the top two sensors indicates that most of the pressure pulses arrive at the top sensor first, implying that most originate near the top of the water column. The average arrival time difference between the top two transducers is $\sim 0.002 \mathrm{~s} \pm 0.002 \mathrm{~s}$ (i.e., one sample interval), which may constrain the speed of sound in the two-phase water-steam fluid in the geyser to a lower bound of about $1500 \mathrm{~m} / \mathrm{s}$, the speed of sound in water. In this case, the main source of dissipation would be scatter by irregularities in the conduit walls rather than the fluid compressibility.

Figure 12 presents the time record as well as the corresponding Fourier spectra for $5 \mathrm{~s}$ of pressure data recorded at the top and middle pressure transducers. From the time record, it is clear that very little dispersion, if any, is observed as the acoustic signal propagates through the water. However, the dissipation is frequency dependent. As Figure 12 demonstrates, high frequencies $(>10 \mathrm{~Hz})$ lose energy while propagating from the top to the middle transducer compared to lower frequencies. A mechanism that might have the above characteristics is the effect of viscous forces felt by a cloud of bubbles under compression. In this case, the fluid viscosity will play an important role as liquid is squeezed between the bubbles during expansion and compression of the cloud. For a speed of sound of $\sim 20 \mathrm{~m} / \mathrm{s}$, the wavelength at $20 \mathrm{~Hz}$ is $\sim 1 \mathrm{~m}$. For pure water at $1 \mathrm{~Hz}$, the wavelength is $1500 \mathrm{~m}$. If the bubble cloud is near the top of the water column at the time of collapse, then wavelengths of the order of the cloud dimension will be strongly dissipated within that region, whereas wavelengths several times longer than the bubbly zone will be dominated by the portion of fluid in the column where the volume fraction of steam is significantly lower.

\subsection{Event Rate}

Several dynamic processes can cause periodic pulsation in a superheated pipe full of coolant, as was discussed in numerous nuclear engineering studies [Boure et al., 1972]. Such regular instabilities are generated
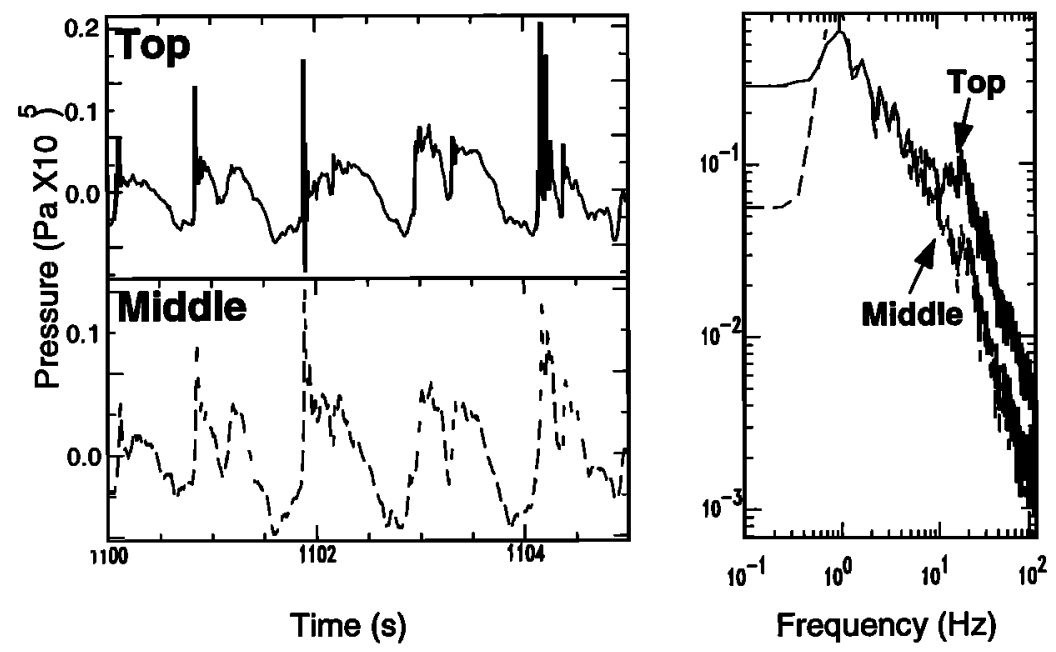

Figure 12. A 5-s-long time window of the top (solid line) and middle (dashed line) pressure sensors and their corresponding Fourier amplitude spectra. Note the difference in spectra at frequencies above $10 \mathrm{~Hz}$. 
when the fluid in the system fluctuates between two thermodynamic states, i.e., when a small thermodynamic perturbation can result in an instantaneous phase change. The excitation of this type of two-phase instabilities is strongly dependent on the heat and mass flux in and out of the system and the geometry of the system.

In this section, the interaction between these parameters, and their influence on the event rate in the water column, will be discussed. The uncertainties about the dynamic effects of the highly turbulent Old Faithful make any attempt to obtain a deterministic model for the event rate a highly complicated, poorly constrained task. Therefore, rather than provide a detailed description of the dynamics of the instabilities, we treat the problem as quasi-static with the following assumptions:

1. The fluid is well mixed (i.e., isothermal.)

2. Each pulse is a cooling event in which a quantum of heat is released.

3 . The rate of occurrence of pulses is solely dependent on the heating rate of the fluid in the conduit, i.e., a pressure pulse corresponding to a cooling event occurs when the temperature in the fluid reaches a certain threshold of supersaturation.

The second and third assumptions need further clarification. We assume that each collapse is the product of an impulsive steam release which occurs as the water column reaches a specific threshold temperature and thus cools the water column instantaneously by a quantum of heat. As heat and water are continuously pumped into the system, the temperature rises again to the threshold temperature and the process repeats itself. When the time interval between pulses is short compared to the time between eruptions, the rate of occurrence of pulses (cooling events) is proportional to the time derivative of the temperature history of the water column.

This calculation should be treated as a thought experiment rather than an attempt to exactly model the dynamic behavior of the geyser. The advantage of these assumptions is that the fluid in the conduit is a well-defined control volume whose temperature history is completely determined by the amount of heat per unit mass, while no consideration of dynamic processes within the control volume, such as heat or mass transport, is necessary. These assumptions are simplistic, as temperature gradients are known to exist in the column, the pulse amplitudes fluctuate, and dynamic constraints on the pulse rate are likely to exist. However, as will be demonstrated, significant insight into the heating process of the water column can be gained with the above assumptions, as simple as they might be.

3.2.1. Filling rate. Consider the system displayed in Figure 13. At time $t_{0}$ a valve is released, and the fluid flows through the thin pipes and fills the larger pipe. We assume that the tank volume is sufficiently large compared to the volume of the pipe, so

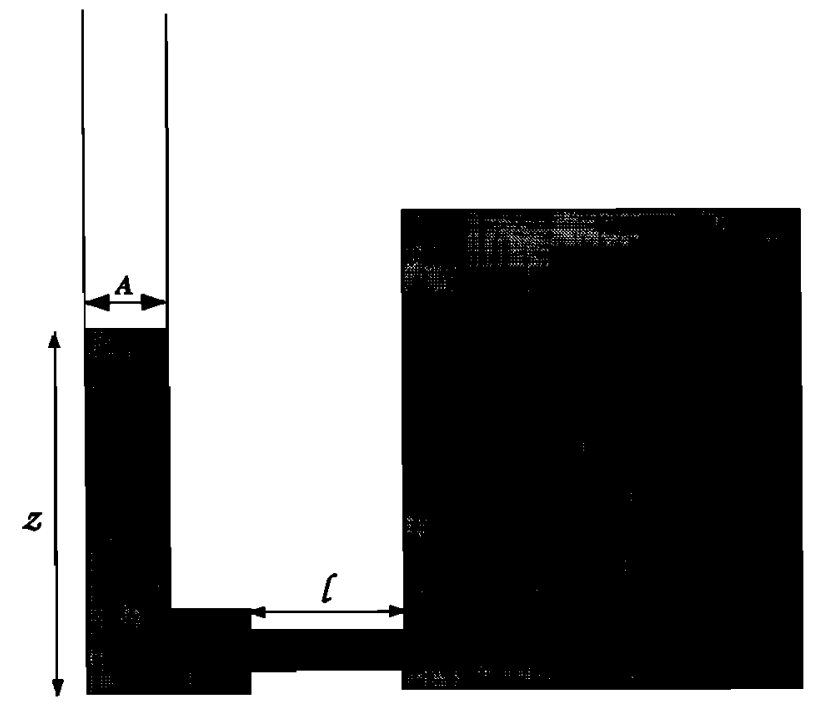

Figure 13. A schematic diagram of a filling model. A tank with liquid of density $\rho$ at pressure $P_{0}$ is connected to a pipe of cross section area $A$, through a network of $n$ pipes of radius $a$ and length $l$.

that the forcing pressure stays $P_{0}$ for the duration of the filling process. First we treat the time history of the water rise in the pipe. The average velocity $u_{a v}$ in one of the $n$ thin pipes of radius $a$ and length $l$, assuming that inertial forces are negligible (i.e., that the flow through the pipe is Poisseuille), is

$$
u_{a v}=\frac{P_{0}-\rho g z}{l} \frac{a^{2}}{8} \frac{1}{\mu}
$$

where $\rho$ is the fluid density, $\mu$ is the viscosity of the fluid $(\mu=\rho \nu)$, and $P_{0}$ is the driving pressure. From conservation of mass, we get

$$
u_{a v}=\dot{z} \frac{A}{n \pi a^{2}}
$$

where $A$ is the pipe (geyser) cross section area and $\dot{z}$ is the time derivative of the water level in the big pipe. Combining equations (7) and (8), the equation of motion for $z$ is

$$
P_{0}-\rho g z-\alpha \dot{z}=0
$$

where $\alpha$ (the constant determining the duration of the filling process) is

$$
\alpha=\frac{8 A \mu l}{n \pi a^{4}}
$$

The above discussion can be similarly formulated in terms of the medium's permeability, $\kappa$. Using Darcy's law for fluid flow in porous media,

$$
\dot{z}=\frac{\kappa}{\mu} \frac{\Delta p}{l}
$$

where $\Delta p=P_{0}-\rho g$. Thus the relationship between the parameter $\alpha$ and the medium permeability $\kappa$ is 


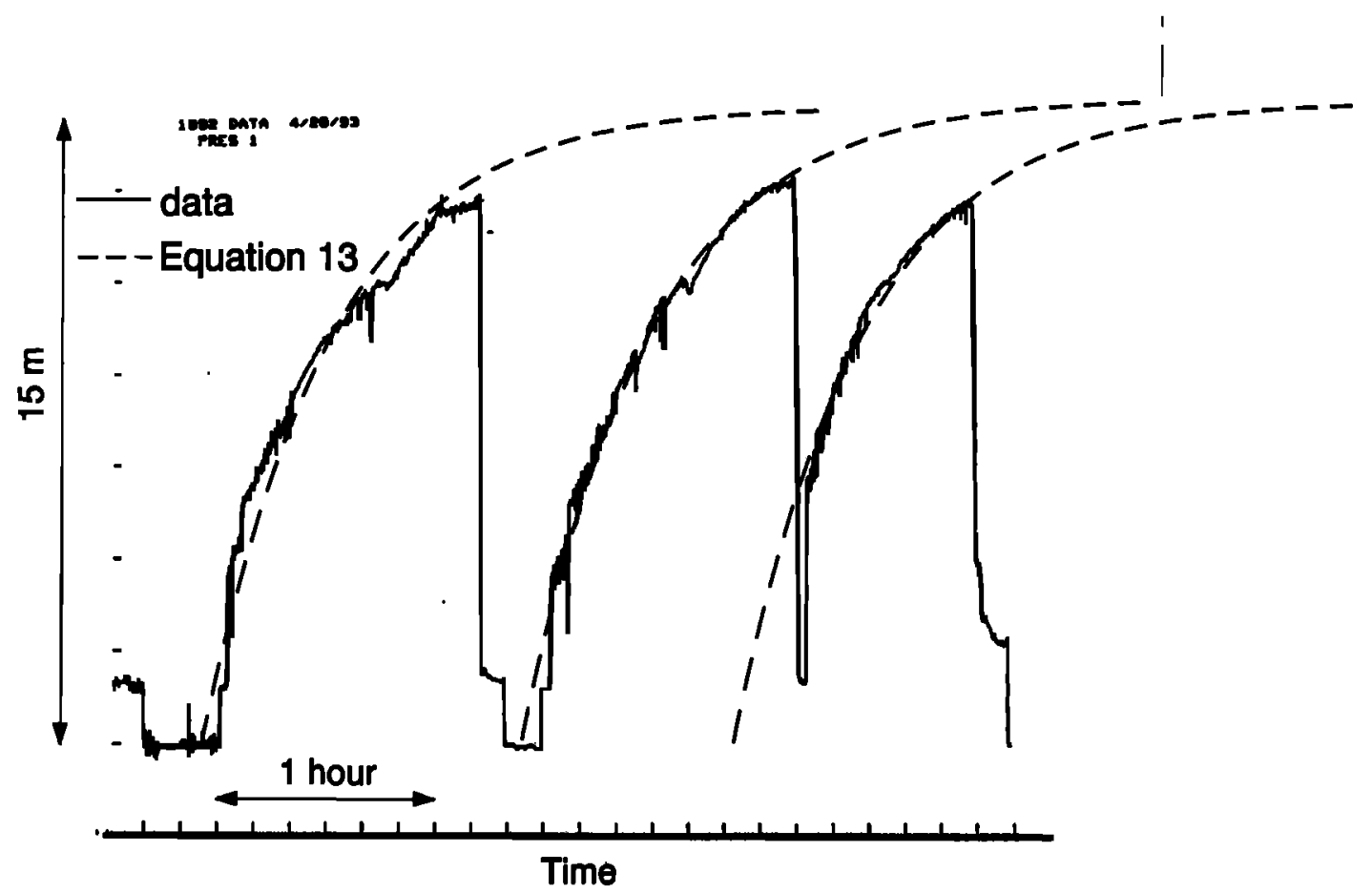

Figure 14. Pressure data (1 sample per second) obtained by J. Westphal, S. Kieffer and R. Hutchinson in 1993, and a calculated filling curve (dashed line) corresponding to $\alpha=2 \times 10^{-7}$ $\mathrm{kg} / \mathrm{m}^{2}$ in equation (13). The model described by Equation 13 obtains a good fit to the data.

$$
\alpha=-\frac{\mu l}{\kappa}
$$

Integrating equation (9), we get

$$
z(t)=\frac{P_{0}}{\rho g}\left[1+C e^{-\left(\frac{\rho g}{\alpha}\right) t}\right]
$$

where $C$ is a constant of integration determined from the initial conditions. For the initial condition $z(t=$ $0)=0, C=-1$.

Comparing the timescales of the calculated column height to the pressure history measurement obtained by J. Westphal et al. (written communication, 1993) (Figure 14), we find that $\alpha=2 \times 10^{7} \mathrm{~kg} / \mathrm{m}^{2}$ s yields good fit to the data.

3.2.2. Heating. The temperature of the water as a function of time can be calculated by assuming a constant heat source. Thus the total of heat $Q$ pumped into the water column at time $t$ is

$$
Q=\int_{t_{0}}^{t} q A(\tau) d \tau
$$

where $q$ is the heat flux and $A(t)$ is the surface area through which heat flows into the water column. The temperature is then calculated using the specific heat at constant pressure $C_{p}$ (for water, $C_{p}=4187 \mathrm{~J} / \mathrm{kg} \mathrm{K}$ )

$$
\Delta h(t)=C_{p}\left(T-T_{0}\right)=\frac{Q(t)}{M(t)}
$$

where $h$ is the enthalpy of the water, $T_{0}$ is the temper- ature of the water entering the system, and $M(t)$ is the total mass in the column as a function of time. In our model,

$$
M(t)=\rho A \int_{t_{0}}^{t} \dot{z} d t=\rho A\left[z(t)-z_{0}\right]
$$

If we assume the heat flows in through the bottom of the cylinder only, then

$$
Q(t)=q A\left(t-t_{0}\right)
$$

Combining equations (15), (16), and (17),

$$
T(t)=T_{0}+\frac{q\left(t-t_{0}\right)}{C_{p} \rho\left[z(t)-z_{0}\right]}
$$

Rinehart [1980] estimated the heat flux $q$ at $5 \times 10^{5}$ $\mathrm{J} / \mathrm{m}^{2} \mathrm{~s}$, which will be used in the following calculations. Rinehart [1980] assumed that the volume of water ejected from Old Faithful during an eruption $(\sim 50$ $\mathrm{m}^{3}$ ) is heated during one eruption cycle ( 1 hour) from groundwater temperature of $5^{\circ} \mathrm{C}$ to $112^{\circ} \mathrm{C}$, the temperature of the water ejected out of Old Faithful. The assumption that the water heats from $5^{\circ} \mathrm{C}$ to $112^{\circ} \mathrm{C}$ during one cycle is probably a gross overestimation since the water might very well be in the system already and preheated to some temperature between the above limits. On the other hand, the system is losing heat by means other than an eruption via the numerous cooling events.

Figure 15a displays the temperature history given by 

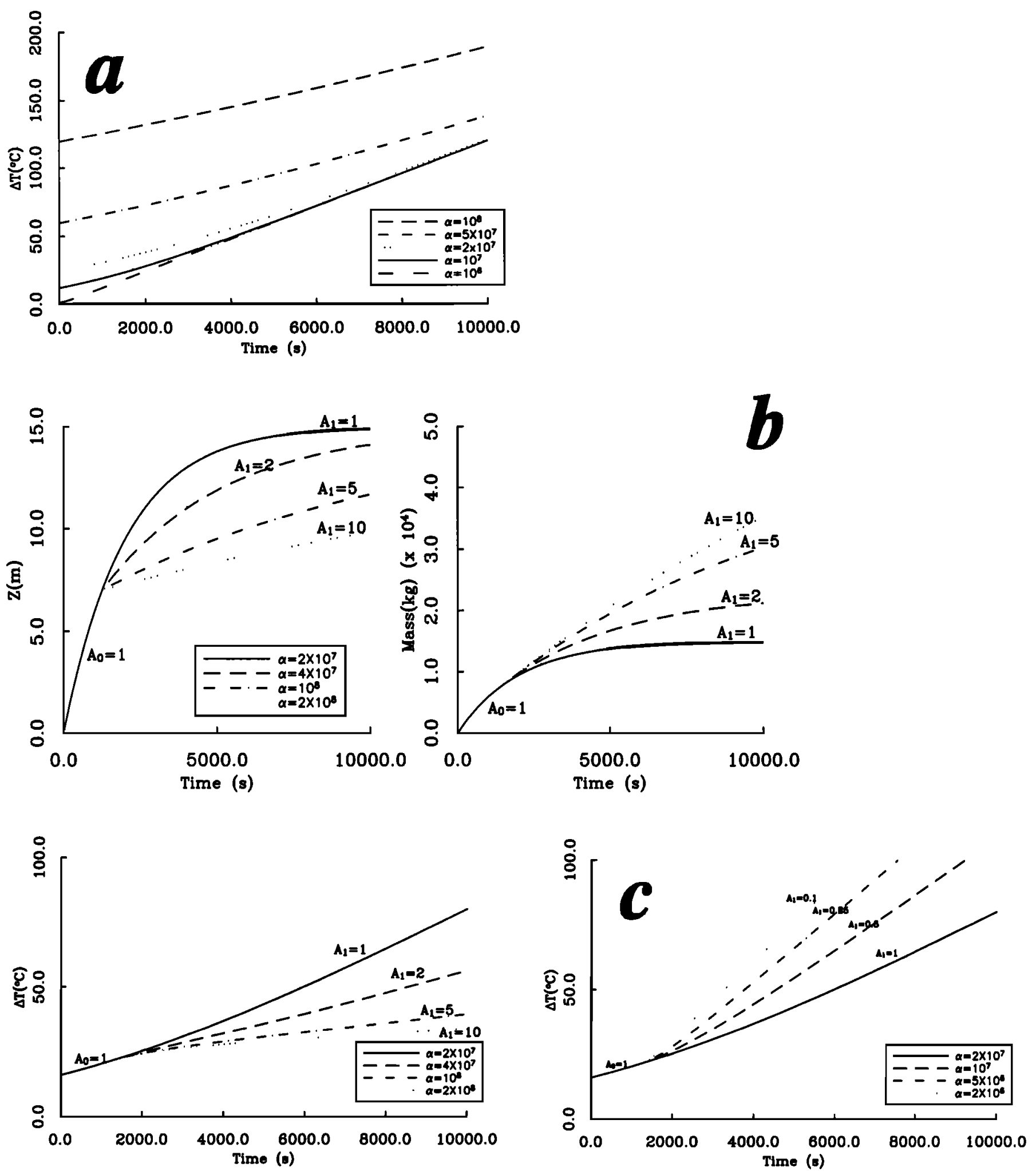

Figure 15. (a) Temperature history for a model heated from below. The slower the filling rate (higher values of $\alpha$ ), the larger the temperature rise per unit time. For the same reason, the curves for smaller values of $\alpha$ approach linearity faster. The initial conditions in all the calculations assume that there is no mass in the pipe at $t=0$ and that the water flowing in is at $0^{\circ} \mathrm{C}$. It is also assumed that the heating of every unit mass is instantaneous, causing a temperature jump at time $t=t_{0}+d t$. Therefore the fluid temperature at infinitesimally short time after $t_{0}=0$ will differ significantly between processes of different heat and mass fluxes. (b) Heating history, water level, and total mass as a function of time, for a pipe widening at $z_{1}=7 \mathrm{~m}$. Different curves describe the behavior for different cross section area $A_{1}$. Note that $\alpha$ is proportional to $A_{1}$. (c) Heating history of a pipe narrowing at $z_{1}=7 \mathrm{~m}$. 


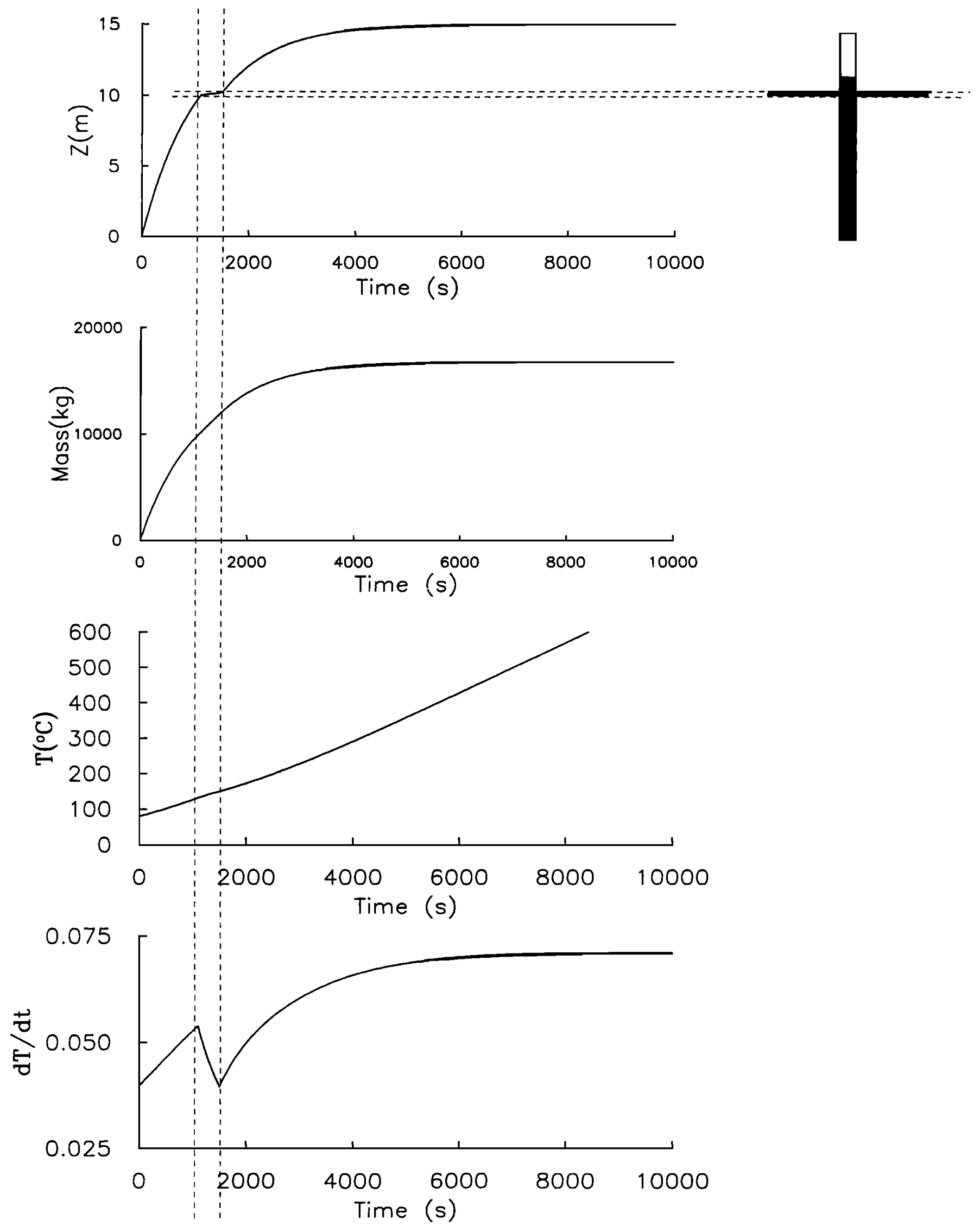

Figure 16. A pipe of cross sectional area $A_{1}=1 \mathrm{~m}^{2}$ opening at $z_{1}=10 \mathrm{~m}$ to $A_{2}=10 \mathrm{~m}^{2}$, and narrowing back to $A_{1}$ at $z_{2}=10.2 \mathrm{~m}$. Shown in the figure are the water-column height $Z(\mathrm{~m})$, the cumulative mass, the heating history, and the derivative of the heating history which determines the rate of events. 
equation (18) using a heat flux $q=5 \times 10^{6} \mathrm{~J} / \mathrm{m}^{2} \mathrm{~s}$. From equations (14) and (15) and from Figure 14, it is apparent that the temperature rise in the column will asymptotically become linear, as the water level approaches its hydrostatic equilibrium level.

3.2.3. Conduit geometry. The "heating from below" model results in a heating curve that is approximately linear in the timescale of $\sim 1$ hour in which we are interested. This implies that the rate of occurrence of heat loss events, which we assume to be proportional to the heating rate, is also linear. Since our observation is that there are at least two regimes of event rates (Figure 4), a constant cross-sectional pipe model cannot explain the observed change in event rate. A possible model that can account for the observed rate change is that of a pipe with a (sudden) change of diameter. It has been observed that such a change does occur in Old Faithful. According to Birch and Kennedy [1972] and Hutchinson et al. [1997], there is an area of widening at about the $10 \mathrm{~m}$ depth from the top of the hole, whose extent is not known.

Assume that a pipe has a cross-section area $A_{0}$ that opens at some depth $z_{1}$ to a cross-section area $A_{1}>A_{0}$. Since the water level rise $\dot{z}$ is slow, we can regard the time when the water level reaches the widened conduit at $Z_{1}$, as a starting point for a new filling process of a pipe of cross section $A_{1}$, but now with a new initial condition $z(t=0)=z_{1}$ which results in a constant $C=z_{1}\left(\rho g / P_{0}\right)-1$ in equation (13). Therefore the temperature is given by

$$
T=T_{0}+\frac{1}{C_{p}} \frac{Q_{0}+q_{0} A_{0} t}{M_{0}+\rho A_{1}\left[z(t)-z_{1}\right]}
$$

where $Q_{0}$ and $M_{0}$ are the heat and mass accumulated in the narrow (cross section $A_{0}$ ) pipe, respectively.

This result is shown in Figure $15 \mathrm{~b}$ with the corresponding water level rise and cumulative mass. As soon as the water level reaches the $z_{1}$ level, the mass flux of fluid into the pipe increases, causing the temperature rise or, equivalently, the cooling-event rate to go down. The wider the pipe, the faster cold water is pushed into the system and the slower the heating rate. This effect can be understood if we consider the limiting case when at $z_{1}$ the pipe opens indefinitely, causing a constant mass flux. Since we assume constant heat flux, the mass of water will asymptotically approach constant temperature. This in turn will cause the cooling events to cease altogether. Similarly, if the pipe narrows at the $z_{1}$ level, then the mass flux goes down and the heating rate increases (Figure $15 \mathrm{c}$ ).

Figure 16 illustrates the behavior of a pipe with a wide section in the middle. The actual temperaturetime curve should appear as a "saw-tooth" shaped curve where every time the temperature reaches the threshold temperature, it drops by an amount corresponding to one heat quantum released by one bubble collapse and heats again to the threshold level following the slope of the heating curve at that instant. As long as the interval between pulses is short compared with the interval between geyser eruptions, as is the case at Old Faithful, the number of "saw-teeth" per unit time will be proportional to the time derivative of the temperature. Figure 16 displays the unperturbed temperature history and its time derivative. It is suggested by this model that the temperature time derivative, and thus the event rate, would drop dramatically when the water level reaches the wide section of the conduit. This calculation illustrates the importance of the role played by the geometry of the pipe in regulating the intensity of the activity.

\section{Conclusions}

The source of continuous harmonic tremor at Old Faithful Geyser can be modeled by discrete bubble collapse events occurring near the top of the superheated water column, each generating a seismic signal. The number of seismic events per unit time, or the intensity of the tremor, is determined by the rate at which bubbles collapse, which in turn depends on the heat and mass flux and is strongly controlled by the geometry of the conduit.

A bubble collapse model with pressure difference $\Delta P=$ $0.3 \times 10^{5} \mathrm{~Pa}$, residual internal gas pressure $P_{g_{0}}=$ $0.2 \times 10^{5} \mathrm{~Pa}$, and an effective viscosity $\nu_{E}=0.04 \mathrm{~m}^{2} / \mathrm{s}$, which implies a bubble radius, $R_{0} \sim 5 \mathrm{~cm}$, is in agreement with our pressure measurements and is consistent with the measured conduit's narrowest spot, $0.1 \mathrm{~m}$ in diameter. Although at this point we cannot identify any damping mechanism which can fully account for the large effective viscosity needed to match the observations, the strong frequency-dependent dissipation suggests a zone of bubbly liquid at the top of the water column in which high frequencies are attenuated and which does not affect longer periods as much.

A constant heat flux from below and a filling process driven by pressure equalization between the aquifer and the conduit through a porous medium give rise to a heating rate which asymptotically approaches a linear temperature increase as the water column exponentially nears its equilibrium level. Neglecting dynamic effects, when the event rate is solely dependent on the heating rate, the time-dependent event intensity can be modulated by widening and narrowing of the conduit, thus controlling the mass flux and consequently the heating rate and the event rate. The modulation of tremor intensity by simple geometric variations may be signifcant in the context of volcanic tremor. This result suggests that an apparent relaxation in tremor intensity does not necessarily mean a pause in the geothermal activity.

Acknowledgments. The authors wish to thank Yellowstone National Park Authorities, the Science Office, and the late Park Geologist Rick Hutchinson for invaluable help in carrying out the experiments. Doug Dreger at UC Berkeley; Blair Zajac, Craig Scrivner, Timothy Melbourne, and 
Miriam Jackson at Caltech; John Holt at JPL; Alessandro Pino at the Istituto Nazionale di Geofisica, Italy, for hard work in the laboratory and in the field; Jim Westphal and Joe Shepherd at Caltech for shared expertise in the design of the probe; and Susan Kieffer for help in planning and analysis of the 1991 experiment. Special thanks to Stan Cincera, Dave Johnson, Wayne Miller, Jose Nunez-Anzueto, Vick Nenow, and Bob Taylor at Caltech for invaluable technical support. We would also like to thank the reviewers for a thorough, constructive critique. Contribution 8492 , Division of Geology and Planetary Sciences, California Institute of Technology.

\section{References}

Birch, F., and G.C. Kennedy, Notes on Geyser temperatures in Iceland and Yellowstone National Park, in Flow and Fracture of Rocks, Geophys. Monogr. Ser., vol. 16, edited by $\mathrm{H}$. C. Heart et al., pp. 329-336, AGU, Washington, D.C., 1972.

Blake, W. K., Mechanics of Flow Induced Sound and Vibrations, vol. 1, Chap. 6, p. 404, Academic, San Diego, Calif., 1986.

Boure, J. A., A. E. Bergels, and L.S. Tong, Review of twophase flow instability, Nucl. Eng. Design, 25, 165-192, 1972.

Chapman, R. B., and M. S. Plesset, Thermal effects in the free oscillation of gas bubbles, J. Basic Eng., 373-376, 1971.

Griffith, P., Geysering in fluid filled lines, paper presented at ASME-AIChE Heat Transfer Conference and Exhibit, Am. Soc. of Mech. Eng., Houston Tex., 1962.

Hutchinson R. A., J. A. Westphal, and S. W. Kieffer, In situ observations of Old Faithful Geyser, Geology, 25 (10), 875-878, 1997.
Kedar, S., B. Sturtevant, and H. Kanamori, The origin of harmonic tremor at Old Faithful Geyser, Nature, 379, 708-711, 1996.

Kieffer, S. W., Sound speed in liquid-gas mixtures: Waterair and water-steam, J. Geophys. Res., 82, 2895-2904, 1977.

Kieffer, S., Seismicity of Old Faithful Geyser: An isolated source of geothermal noise and possible analogue of volcanic tremor, J. Volcanol. Geotherm. Res., 22, 59-96, 1984.

Leet, R. C., Saturated and subcooled hydrothermal boiling in groundwater flow channels as a source of harmonic tremor, J. Geophys. Res., 93, 4835-4849, 1988.

Plesset, M. S., The dynamics of cavitation bubbles, ASME J. Appl. Mech., 16, 228-231, 1949.

Rayleigh, On the pressure developed in a liquid during the collapse of a spherical bubble, Philos. Mag., 34, 94-98, 1917.

Rinehart, J. S., Earth tremors generated by Old Faithful Geyser, Science, 150, 494-496, 1965.

Rinehart, J. S., Geophysical study of geyser action in Yellowstone National Park, J. Geophys. Res., 72(18), 4651-4663, 1967.

Rinehart, J. S., Geysers and Geothermal Energy, SpringerVerlag, New York, 1980.

H. Kanamori and S. Kedar, Seismological Laboratory, 252-21, California Institute of Technology, Pasadena, CA 91125. (e-mail: sharon@gps.caltech.edu)

B. Sturtevant, Graduate Aeronautics Laboratories, California Institute of Technology, Pasadena, CA 91125

(Received November 12, 1997; revised March 17, 1998; accepted May 27, 1998.) 